\title{
Fast quantum noise in the Landau-Zener transition
}

\author{
V. L. Pokrovsky and D. Sun \\ Department of Physics, Texas A\&M University, College Station, Texas 77843, USA
}

(Received 20 March 2007; revised manuscript received 15 June 2007; published 30 July 2007)

\begin{abstract}
We show by direct calculation starting from a microscopic model that the two-state system with timedependent energy levels in the presence of fast quantum noise obeys the master equation. The solution of master equation is found analytically and analyzed in a broad range of parameters. The fast transverse noise affects the transition probability during much longer time (the accumulation time) than the longitudinal one. The action of the fast longitudinal noise is restricted by the shorter Landau-Zener time, the same as in the regular Landau-Zener process. The large ratio of time scales allows solving the Landau-Zener problem with longitudinal noise only, and then solving the same problem with the transverse noise only and matching the two solutions. The correlation of the longitudinal and transverse noise renormalizes the Landau-Zener transition matrix element and can strongly enhance the survival probability, whereas the transverse noise always reduces it. If the noise is fast, its intensity at which the multiquantum processes become essential corresponds to a deeply adiabatic regime. We briefly discuss possible applications of the general theory to the molecular magnets.
\end{abstract}

DOI: 10.1103/PhysRevB.76.024310 PACS number(s): 05.40.Ca, 03.65. - w, 33.80.Be, 32.80. -t

\section{INTRODUCTION}

Landau-Zener (LZ) theory ${ }^{1-3}$ deals with a two-state system, whose levels vary in time by sweeping of some physical parameters and would cross at some moment. Transitions between the two states are induced by the transition matrix element $\Delta$, which in static or adiabatic case causes the level repulsion (avoided crossing according to the WignerNeumann theorem). The LZ theory is one of basic dynamic problems of quantum mechanics. It has a broad range of applications from the collision theory and quantum chemistry to disordered solids and molecular magnets.

The LZ transitions in noisy environment recently attracted new attention in connection with the problem of the qubit decoherence. ${ }^{4,5}$ New experimental realization of qubits ${ }^{6}$ gives rise again to the question as to what extent it is possible to minimize the decoherence simultaneously maintaining sufficiently strong coupling to the external signal.

The problem of the LZ transition in noisy environment has a relatively long history. One of the first considerations based on ideas of stochastic trajectories belongs to Kasunoki. ${ }^{7}$ In the pioneering work, ${ }^{8}$ Kayanuma calculated the transition amplitude in the presence of a fast transverse Gaussian classical noise with a specific (exponential) twotime correlation function. ${ }^{9}$ This solution was simplified and extended to general shape of correlation function by Pokrovsky and Sinitsyn. ${ }^{11}$ In the same work, a situation in which the transitions are produced by noise as well as by regular Hamiltonian was considered. Pokrovsky and Scheidl ${ }^{12}$ calculated the two-time correlation function of the transition probabilities for the LZ system subject to a fast classical transverse noise. Longitudinal noise was considered by Kayanuma, ${ }^{10}$ who proved that strong fast longitudinal noise enhances the nonadiabaticity, and the transition probability is given by a formula different from the LZ formula, while slow longitudinal noise does not change the LZ transition probability. Gefen et al..$^{13}$ and Ao and Rammer ${ }^{14}$ considered more wide range of parameters and found the situations in which the noise changes the transition probability. In that work, ${ }^{14}$ a rather detailed analysis of different limiting cases of temperature, coupling to the phonon bath, its spectral width, and sweeping rate was presented. There occurred a controversy between Refs. 13 and 14. Generally, there is no complete agreement between different authors on what happens in the adiabatic regime (very slow sweeping) in the presence of the longitudinal noise. Motivated by this disagreement, Kayanuma and Nakayama performed a comprehensive analytical and numerical study of the LZ transition in the presence of longitudinal noise. ${ }^{15}$ In particular, they obtained a formula for the case of strong decoherence, which is valid in both low-temperature and high-temperature limits. In all these works, the quantum nature of the longitudinal noise was taken into account.

Despite significant progress, a complete theory of the LZ transition in noisy environment still does not exist. Theoretical works considered either quantum longitudinal noise with transitions originated from the regular transition matrix element or the classical transverse noise. Quite recently, Wubs et al. ${ }^{16}$ have found a beautiful exact formula for the transition probability of the two-state system interacting with the phonon bath at zero temperature. ${ }^{17}$ The noise had both longitudinal and transverse components. Their correlation and quantum nature were substantial. No limitations to the noise strength and spectral width were assumed. However, the limitation of zero temperature (phonon bath is in the ground state) does not allow one to extend these results to more realistic situations.

The purpose of this paper is to present a theoretical description of the LZ system subject to a fast quantum noise, which has both transverse and longitudinal components. It is not yet a complete theory, since it does not cover slow and intermediate noise, but in its range of applicability, it allows us to understand clearly all relevant physical regimes and phenomena. We will show that, due to the fastness of the noise, the LZ transition in the presence of the longitudinal noise and the transitions due to the transverse noise are separated in time, whereas the correlation between the transverse 
and longitudinal noise leads to a renormalization of the regular transition matrix element in the LZ Hamiltonian. For a moderately strong transverse noise, we derive master equations governing the population of the two states and study their solution. If the transverse noise is strong and also fast, the two-state system falls into the adiabatic regime. The population of levels comes to the equilibrium with the spin bath if the bath is in the state of thermal equilibrium. We argue that a very strong noise is classical and adiabatic. In this situation, as it was shown in Ref. 11, the populations of the two levels are equal.

The plan of the paper is as follows. In Sec. II, we introduce the Hamiltonian and characterize the noise. In Sec. III, we present simple heuristic arguments resulting in master equations. In Sec. IV, we derive the master equations starting from microscopic Hamiltonian for the case of the transverse noise only and zero LZ transition matrix element. In Sec. V, we derive the renormalization of the regular transition matrix element due to correlation of longitudinal and transverse noise. In Sec. VI, we analyze the influence of the longitudinal noise. In Sec. VII, we find the solution of master equation and study it. In Sec. VIII, we match the solution of the master equation with the solution of the LZ problem without transverse noise. Section IX contains discussion and conclusions. Here, we compare our theory with that by Wubs et $a l .{ }^{16} \mathrm{We}$ briefly analyze possible applications of our theory to molecular magnets.

\section{STATEMENT OF THE PROBLEM}

We consider a two-state system interacting with a noisy environment. The latter is a large system (bath) with a stationary density matrix. We neglect the influence of the LZ transitions on the state of the bath. For definiteness, we will speak about the phonon bath, though it can include other Boson excitations such as spin waves, excitons, and photons. Then, the total Hamiltonian of the system can be represented as follows:

$$
H=H_{2}+H_{b}+H_{\text {int }} .
$$

The term $\mathrm{H}_{2}$ in Eq. (1) represents the two-state system:

$$
H_{2}=-\frac{\Omega(t)}{2} \sigma_{z}+\Delta \sigma_{x},
$$

where $\sigma_{x}$ and $\sigma_{z}$ are Pauli matrices and $\Omega(t)$ is the timedependent frequency or the energy difference between the so-called diabatic levels turning into zero at $t=0 ; \Delta$ is the regular transition matrix element. If $t$ would be not time but some parameter of the Hamiltonian, then nonzero $\Delta$ provides repulsion of the adiabatic levels (the Wigner-Neumann theorem on avoided level crossing). For brevity, we will further call the regular transition matrix element $\Delta$ the LZ gap. Usually, the linear approximation for the frequency $\Omega(t)=\dot{\Omega} t$ proposed by Landau and Zener is acceptable, but sometimes, it is necessary to go beyond this approximation. Namely, in real experiment, the sweeping of $\Omega(t)$ stops at some finite value, which is not necessarily large in the frequency scale of the problem. Therefore, we will keep the notation $\Omega(t)$ throughout this paper. The term $H_{b}$ in Eq. (1) is the phonon bath Hamiltonian:

$$
H_{b}=\sum_{\mathbf{q}} \omega_{\mathbf{q}} b_{\mathbf{q}}^{\dagger} b_{\mathbf{q}},
$$

where $b_{\mathbf{q}}$ and $b_{\mathbf{q}}^{\dagger}$ are the operators of the phonon annihilation and creation; $\omega_{\mathbf{q}}$ are the phonon frequencies and $\mathbf{q}$ are their momenta. The interaction Hamiltonian reads

$$
H_{\text {int }}=u_{\|} \sigma_{z}+u_{\perp} \sigma_{x} .
$$

The Hermitian operators $u_{\|}$and $u_{\perp}$ responsible for the longitudinal and transverse noise depend linearly on the phonon operators. Each of them is a sum of two Hermitian conjugated operators containing either phonon annihilation or creation operators only:

$$
u_{\alpha}=\eta_{\alpha}+\eta_{\alpha}^{\dagger}, \quad \eta_{\alpha}=\frac{1}{\sqrt{V}} \sum_{\mathbf{q}} g_{a}(\mathbf{q}) b_{\mathbf{q}}, \quad \alpha=\|, \perp,
$$

where $g_{a}(\mathbf{q})$ are complex coupling amplitudes and $V$ is the volume of the system supporting phonons. Quantum character of the noise manifests itself in noncommutativity of operators $\eta_{\alpha}$ and $\eta_{\alpha}^{\dagger}$. The problem consists in calculation of transition and surviving probabilities for the two-state system at a fixed noise density matrix. In the absence of the noise, the transition amplitudes constitute the LZ transition matrix belonging to the $\mathrm{SU}(2)$ group and depending on the dimensionless LZ parameter $\gamma_{L Z}=\Delta^{2} / \dot{\Omega}$ :

$$
\begin{aligned}
& T_{L Z}=\left(\begin{array}{cc}
\alpha & \beta \\
-\beta^{*} & \alpha^{*}
\end{array}\right), \\
& \alpha=e^{-\pi \gamma_{L Z}}, \quad \beta=-\frac{\sqrt{2 \pi} \exp \left(-\frac{\pi \gamma_{L Z}}{2}+i \frac{\pi}{4}\right)}{\sqrt{\gamma_{L Z}} \Gamma\left(-i \gamma_{L Z}\right)} .
\end{aligned}
$$

If $\gamma_{L Z}$ is small, the system with the probability close to 1 remains in initial diabatic state; if $\gamma_{L Z}$ is large, the system with probability close to 1 proceeds along the adiabatic state, i.e., changes the initial diabatic state to the alternative one. The characteristic time necessary for the LZ transition is $\tau_{L Z}=\max \left(\Delta / \dot{\Omega}, \dot{\Omega}^{-1 / 2}\right)$.

Since the noise is Gaussian, its influence on the two-state system is completely described by the noise correlation functions

$$
\begin{gathered}
\left\langle\eta_{\alpha}(t) \eta_{\beta}^{\dagger}\left(t^{\prime}\right)\right\rangle=\frac{1}{V} \sum_{\mathbf{q}} g_{\alpha}(\mathbf{q}) g_{\beta}^{*}(\mathbf{q})\left(n_{\mathbf{q}}+1\right) e^{i \omega_{\mathbf{q}}\left(t^{\prime}-t\right)}, \\
\left\langle\eta_{\alpha}^{\dagger}(t) \eta_{\beta}\left(t^{\prime}\right)\right\rangle=\frac{1}{V} \sum_{\mathbf{q}} g_{\alpha}(\mathbf{q}) g_{\beta}^{*}(\mathbf{q}) n_{\mathbf{q}} e^{i \omega_{\mathbf{q}}\left(t-t^{\prime}\right)} .
\end{gathered}
$$

Here, $n_{\mathbf{q}}=\left\langle b_{\mathbf{q}}^{\dagger} b_{\mathbf{q}}\right\rangle$ are the average phonon occupation numbers and $\langle\ldots\rangle$ means averaging over the phonon bath ensemble, not necessarily in thermal equilibrium. The Fourier components of the correlation functions (7) and (8) read

$$
\left\langle\eta_{\alpha} \eta_{\beta}^{\dagger}\right\rangle_{\omega}=\frac{2 \pi}{V} \sum_{\mathbf{q}} g_{\alpha}(\mathbf{q}) g_{\beta}^{*}(\mathbf{q})\left(n_{\mathbf{q}}+1\right) \delta\left(\omega-\omega_{\mathbf{q}}\right),
$$


TABLE I. Different time scales and definitions.

\begin{tabular}{lccc}
\hline \hline$\tau_{n}$ & $\tau_{L Z}$ & $\tau_{a c c}$ & $\tau_{r \alpha}$ \\
\hline$\tau_{n}=1 / \Delta \omega$ & $\tau_{L Z}=\max \left(\Delta / \dot{\Omega}, \dot{\Omega}^{-1 / 2}\right)$ & $\tau_{a c c}=\left(\dot{\Omega} \tau_{n}\right)^{-1}$ & $\tau_{r \alpha}=\left(\left\langle u_{\alpha}^{2}(t)\right\rangle \tau_{n}\right)^{-1}$ \\
Fast & Weak & Moderate & Strong \\
$\tau_{n} \ll \min \left(\dot{\Omega}^{-1 / 2}, \Delta^{-1}\right)$ & $\left\langle u_{\alpha}^{2}(t)\right\rangle \ll \dot{\Omega}$ & $\dot{\Omega} \leqslant\left\langle u_{\alpha}^{2}(t)\right\rangle \ll \tau_{n}^{-2}$ & $\left\langle u_{\alpha}^{2}(t)\right\rangle \geqslant \tau_{n}^{-2}$ \\
\hline \hline
\end{tabular}

$$
\left\langle\eta_{\alpha}^{\dagger} \eta_{\beta}\right\rangle_{\omega}=\frac{2 \pi}{V} \sum_{\mathbf{q}} g_{\alpha}(\mathbf{q}) g_{\beta}^{*}(\mathbf{q}) n_{\mathbf{q}} \delta\left(\omega+\omega_{\mathbf{q}}\right) .
$$

Note that one of the two correlators contains only positive frequencies, whereas the second one contains only negative frequencies. If the noise is in equilibrium at temperature $T$, the Fourier transforms of correlation functions obey the following simple relation $(\omega>0)$ :

$$
\frac{\left\langle\eta_{\alpha} \eta_{\beta}^{\dagger}\right\rangle_{\omega}}{\left\langle\eta_{\alpha}^{\dagger} \eta_{\beta}\right\rangle_{-\omega}}=e^{\omega / T}
$$

Let us denote $\omega_{g}$ the range of frequencies in which the coupling coefficients $g_{\alpha}(\mathbf{q})$ do not vanish. If the occupation numbers $n_{\mathbf{q}}$ are of the same order of magnitude for all states within this region of frequencies, then $\omega_{g}$ determines the spectral width of the noise $\Delta \omega$. In some cases, for example, at low temperature $T \ll \omega_{g}$, there appears a second, smaller scale of frequency $(T)$. The noise correlation time is $\tau_{n}$ $=1 / \Delta \omega$. It is different for the two correlation functions $\left\langle\eta_{\alpha}(t) \eta_{\beta}^{\dagger}\left(t^{\prime}\right)\right\rangle$ and $\left\langle\eta_{\alpha}^{\dagger}(t) \eta_{\beta}\left(t^{\prime}\right)\right\rangle$ at low temperature and it is equal to $\omega_{g}^{-1}$ for both at high temperature. By definition, the noise is fast if $\tau_{n} \ll \min \left(\dot{\Omega}^{-1 / 2}, \Delta^{-1}\right)$ or $\Delta \omega \gg \max \left(\dot{\Omega}^{1 / 2}, \Delta\right)$. Besides its spectral characteristics, the noise is characterized by its strength. The most natural measure of the noise strength is the average square of its amplitude:

$\left\langle u_{\alpha}^{2}(t)\right\rangle=\left\langle\eta_{\alpha}(t) \eta_{\alpha}^{\dagger}(t)\right\rangle+\left\langle\eta_{\alpha}^{\dagger}(t) \eta_{\alpha}(t)\right\rangle=\frac{1}{V} \sum_{\mathbf{q}}\left|g_{\alpha}(\mathbf{q})\right|^{2}\left(2 n_{\mathbf{q}}+1\right)$.

The noise is weak if $\left\langle u_{\alpha}^{2}(t)\right\rangle \ll \dot{\Omega}$. Weak noise can be accounted as a small perturbation to the LZ result. We call the noise moderately strong if it obeys the inequalities $\dot{\Omega}$ $\leqslant\left\langle u_{\alpha}^{2}(t)\right\rangle \ll \tau_{n}^{-2}$. Though for moderately strong noise the perturbation theory is generally invalid at accumulation time scale determined in the next section, we will show that it works during sufficiently small intervals of time still longer than $\tau_{n}$. Most of our results relate to the weak and moderate noise, i.e., for the noise obeying the condition $\left\langle u_{\alpha}^{2}(t)\right\rangle \ll \tau_{n}^{-2}$, which we will call not strong. The noise is called strong if $\left\langle u_{\alpha}^{2}(t)\right\rangle \geqslant \tau_{n}^{-2}$. Note that generally $\dot{\Omega}$ depends on time, so that the noise may be weak or moderate during one time interval and strong during another one. Another time scale, which we call the relaxation time $\tau_{r \alpha}=\left(\left\langle u_{\alpha}^{2}(t)\right\rangle \tau_{n}\right)^{-1}$ (see Ref. 15, where it is called phase relaxation time), is defined by the condition that the probability of finding the system in state 1 or 2 changes significantly. When the noise is strong, the relaxation time becomes less than the noise correlation time $\tau_{n}$.
The definitions of different time scales and their connection to the parameters of the problem are collected in the Table I. It is convenient to introduce dimensionless coupling function $\lambda_{\alpha \mathbf{q}}=\left|g_{\alpha}(\mathbf{q})\right| /\left(\omega_{g} a^{3}\right)$, where $a$ is the lattice constant, $\alpha=\|, \perp$. In condensed matter systems, the values $\lambda_{\alpha \mathbf{q}}$ never become large. Migdal ${ }^{20}$ argued that large coupling constants would lead to the lattice instability and reconstruction. Though his arguments related directly to the electron-phonon coupling, his idea is very general. If $\lambda_{\alpha \mathbf{q}}$ are not large, a large value of $\left\langle u_{\alpha}^{2}(t)\right\rangle \tau_{n}^{2}$ can be reached only if phonon occupation numbers $n_{\mathbf{q}}$ become large. In the equilibrium case, it means that the temperature must be large. Very strong noise is classical, irrespective of its specific density matrix. An analog of the LZ parameter for the noise reads ${ }^{11} \gamma_{n \alpha}=\left\langle u_{\alpha}^{2}(t)\right\rangle / \dot{\Omega}$. If it is small, the noise brings only a small perturbation to the LZ picture; if it is large, the occupation numbers of the two-state system follow adiabatically the instantaneous value of frequency. We will return to this point later.

\section{HEURISTIC APPROACH}

In the previous section, we argued that the LZ gap and longitudinal noise become ineffective for transitions beyond the LZ time interval $\left(-\tau_{L Z}, \tau_{L Z}\right)$. We show later in this section that the transverse noise produces transitions during much longer time interval $\left(-\tau_{a c c}, \tau_{a c c}\right)$, where $\tau_{a c c}=\left(\dot{\Omega} \tau_{n}\right)^{-1}$. Therefore, for $|t| \gg \tau_{L Z}$, it is necessary to solve a simplified problem with $\Delta=0$ and $u_{\|}=0$, so that all transitions are only due to the transverse noise. In this section, we develop a heuristic approach to this simplified problem.

Since $\tau_{n} \ll \dot{\Omega}^{-1 / 2}$, the instantaneous frequency of the twostate system does not change during correlation time of the noise and it can be considered as a constant. Thus, it is possible to calculate the instantaneous rate of transition probability using the standard quantum mechanical technique for transitions between stationary energy levels. If the noise is not strong, the transition probability can be calculated in the first Born approximation. Indeed, according to the Fermi golden rule, the rate of transition probability per unit time before the level crossing reads

$$
p_{1 \rightarrow 2}(t)=2 \pi\left\langle\eta_{\perp}^{\dagger} \eta_{\perp}\right\rangle_{\Omega(t)}
$$

In the framework of the considered model, the next after single-phonon transition is three-phonon transition shown in Fig. 1. Its contribution to the transition probability reads 


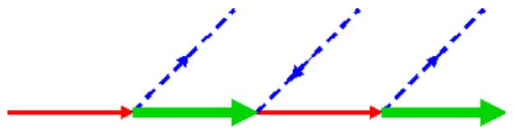

FIG. 1. (Color online) Feynman graph for a three-phonon process. Thin solid lines correspond to state 1, thick solid lines correspond to state 2, and dashed blue lines correspond to phonons.

$$
p_{1 \rightarrow 2}^{(3)}(t)=2 \pi\left\langle\int \frac{d \omega_{1} d \omega_{2}}{(2 \pi)^{2}} \frac{\eta_{\omega_{1}}^{\dagger} \eta_{\omega_{2}}^{\dagger} \eta_{\Omega(t)-\omega_{1}-\omega_{2}}^{\dagger}}{\omega_{1} \omega_{2}} \times \text { H.c. }\right\rangle .
$$

The three-phonon contribution can be neglected if the noise is moderately strong. In the same approximation, it is possible to neglect the correction to the transition frequency due to the interaction with the phonon bath. Thus, the occupation numbers of the diabatic states $N_{1,2}$ at negative time obey the following master equation:

$$
\dot{N}_{1}=2 \pi\left(-\left\langle\eta_{\perp}^{\dagger} \eta_{\perp}\right\rangle_{|-\Omega(t)|} N_{1}+\left\langle\eta_{\perp} \eta_{\perp}^{\dagger}\right\rangle_{|\Omega(t)|} N_{2}\right),
$$

which must be complemented by conservation law $N_{1}+N_{2}$ $=1$. For positive time, Eq. (15) must be modified as follows:

$$
\dot{N}_{1}=2 \pi\left(-\left\langle\eta_{\perp} \eta_{\perp}^{\dagger}\right\rangle_{|\Omega(t)|} N_{1}+\left\langle\eta_{\perp}^{\dagger} \eta_{\perp}\right\rangle_{|-\Omega(t)|} N_{2}\right) .
$$

The noise produces transitions as long as its spectral width exceeds the instantaneous frequency $|\Omega(t)|$. The accumulation time estimated from this requirement is $\tau_{a c c}=\left(\dot{\Omega} t_{n}\right)^{-1} \cdot{ }^{11}$ Since the noise is assumed to be fast, the accumulation time $\tau_{a c c}$ is much longer than the noise correlation time $\tau_{n}$. In real experiment, the sweeping of frequency may stop or saturate before the accumulation time is reached. The master equations enable one to calculate the occupation numbers at any time rather than asymptotically at $t \rightarrow \infty$. The accumulation time is also much longer than the LZ time $\tau_{L Z}$. Therefore, it is possible to neglect the action of the noise during the LZ time interval $\left(-\tau_{L Z}, \tau_{L Z}\right)$ and neglect the LZ gap $\Delta$ beyond this time interval. It means that the actions of the fast transverse noise and of the LZ gap are separated in time as it was shown earlier for classical noise. ${ }^{11}$ The solution of the LZ problem without transverse noise and the noise transition problem with zero LZ gap $\Delta$ should be matched at some intermediate time. It will be done in Sec. VIII.

The action of the fast longitudinal noise is very different from that of the transverse one. The longitudinal noise does not produce transitions in the absence of the LZ gap. Therefore, its action is effectively limited to the LZ time interval. In the next section, we demonstrate that the fast longitudinal noise must be sufficiently strong to produce a substantial change in the LZ transition probability. Namely, it must satisfy the inequality $\left\langle u_{\|}^{2}\right\rangle \gtrsim \dot{\Omega} /\left(\Delta \tau_{n}\right) \gg \dot{\Omega}$. An analogous criterion for the transverse noise is much more liberal: $\left\langle u_{\perp}^{2}\right\rangle \gtrsim \dot{\Omega}$. For a comprehensive analysis of the longitudinal noise action, we refer the reader to Refs. 13-15. Beyond the LZ time interval, the classical longitudinal noise modulates the transverse noise by a factor $\exp \left(-i \int_{t_{0}}^{t} u_{\|} d \tau\right)$. Correlation functions $\left\langle\eta_{\perp}(t) \eta_{\perp}^{\dagger}\left(t^{\prime}\right)\right\rangle$ must be substituted by $\left\langle\eta_{\perp}(t) \eta_{\perp}^{\dagger}\left(t^{\prime}\right)\right.$ $\left.\times \exp \left(-i \int_{{ }^{\prime}}^{t} u \| d \tau\right)\right\rangle$. Neglecting the correlation between longitudinal and transverse noise and employing the Gaussian statistics of the noise, one can express the latter correlator as follows:

$$
\begin{aligned}
& \left\langle\eta_{\perp}(t) \eta_{\perp}^{\dagger}\left(t^{\prime}\right) \exp \left(-i \int_{t^{\prime}}^{t} u_{\|} d \tau\right)\right\rangle \\
& \quad=\left\langle\eta_{\perp}(t) \eta_{\perp}^{\dagger}\left(t^{\prime}\right)\right\rangle \exp \left[-\frac{1}{2} \int_{t^{\prime}}^{t} \int_{t^{\prime}}^{t} d t_{1} d t_{2}\left\langle u_{\|}\left(t_{1}\right) u_{\|}\left(t_{2}\right)\right\rangle\right] .
\end{aligned}
$$

The transverse noise correlator decays rapidly when the modulus of difference $\left|t-t^{\prime}\right|$ exceeds $\tau_{n}$. Therefore, the value in the exponent in the right-hand side of Eq. (17) can be estimated as $\left\langle u_{\|}^{2}\right\rangle \tau_{n}^{2} \ll 1$. This estimate shows that the longitudinal noise can be neglected beyond the LZ time interval. In the next section, we consider this question in more detail.

Though physical arguments of this section leading to the master equation sound convincing, we used an implicit assumption that the quantum coherence is negligibly small. In the next section, we derive the master equation rigorously starting from the microscopic Hamiltonian. This derivation shows that, though quantum coherence is substantial at small time intervals less than $\tau_{n}$, it does not play any role at larger time scale.

\section{DERIVATION OF MASTER EQUATIONS}

In this section, as in the previous one, we consider time interval beyond Landau-Zener one $\left(-\tau_{L Z}, \tau_{L Z}\right)$ and neglect the LZ gap $\Delta$ and longitudinal noise. Our goal is to find the dependence of the occupation numbers $N_{\alpha}(\alpha=1,2)$ on time. The same problem can be formulated as calculation of the average value of projectors $P_{\alpha}=|\alpha\rangle\langle\alpha|=\frac{1}{2}\left(1 \pm \sigma_{z}\right){ }^{10,14} \mathrm{We}$ consider the case $\Delta=0, u_{\|}=0$. The calculation will be performed in the interaction representation with the diagonal time-dependent unperturbed Hamiltonian $H_{0}=-\frac{\Omega(t)}{2} \sigma_{z}+H_{b}$ $=-\frac{\Omega(t)}{2}(|1\rangle\langle 1|-| 2\rangle\langle 2|)+H_{b}$ and the interaction Hamiltonian $V=u_{\perp} \sigma_{x}=u_{\perp}(|1\rangle\langle 2|+| 2\rangle\langle 1|)$. Being transformed to the interaction representation, the interaction Hamiltonian depends on time as follows:

$$
\begin{aligned}
V_{I}(t)= & u_{\perp}(t)\left[|1\rangle\langle 2| \exp \left(-i \int_{t_{0}}^{t} \Omega(\tau) d \tau\right)\right. \\
& \left.+|2\rangle\langle 1| \exp \left(i \int_{t_{0}}^{t} \Omega(\tau) d \tau\right)\right]
\end{aligned}
$$

where

$$
u_{\perp}(t)=e^{i H_{b}\left(t-t_{0}\right)} u_{\perp}\left(t_{0}\right) e^{-i H_{b}\left(t-t_{0}\right)} .
$$

The time-dependent occupation numbers can be expressed in terms of the evolution operator $U_{I}\left(t, t_{0}\right)$ in the interaction representation:

$$
N_{\alpha}(t)=\operatorname{Tr}\left[\rho_{0} U_{I}^{-1}\left(t, t_{0}\right) P_{\alpha} U_{I}\left(t, t_{0}\right)\right],
$$

where $\rho_{0}$ is the initial density matrix which is the direct product of two independent density matrices $\rho_{0}=\rho_{2} \rho_{b}$, where 


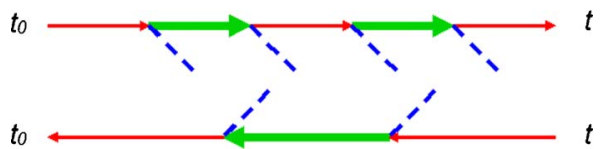

FIG. 2. (Color online) An example of a term in the perturbation theory. Points correspond to vertices $V_{I}\left(t_{j}\right)$.

the first factor is the density matrix of the two-state system and the second one is the same for the bath. For calculation of these averages we employ a simplified version of the Keldysh-Schwinger technique, ${ }^{21,22}$ used already for a similar purpose in Refs. 10, 14, and 15. Each of the two evolution operators is presented as a series of time ordered integrals. A general term of such an expansion contains a product of two multiple time integrals. With each time variable $t_{k}$, a vertex $V_{I}\left(t_{k}\right)$ is associated. The product of vertices is ordered chronologically in $U_{I}\left(t, t_{0}\right)$ and antichronologically in $U_{I}^{-1}\left(t, t_{0}\right)$. All operators of $V_{I}\left(t_{k}\right)$ belonging to $U_{I}^{-1}\left(t, t_{0}\right)$ are located on the left ("later") than corresponding operators belonging to $U_{I}\left(t, t_{0}\right)$. A particular contribution is graphically depicted in Fig. 2 before the averaging over phonon bath is performed. It consists of two lines both starting at $t_{0}$ and ending at $t$. The upper line symbolizes $U_{I}\left(t, t_{0}\right)$ and the lower one symbolizes $U_{I}^{-1}\left(t, t_{0}\right)$. Vertices on these lines correspond to the operators $V_{I}\left(t_{k}\right)$. Each vertex contains one phonon operator $u\left(t_{k}\right)$ and changes the state of the two-state system. The presence of the projection operator $P_{\alpha}$ in Eq. (20) implies that the state closest to the final time $t$ on both lines must be $|\alpha\rangle$. Applying the Wick's rule for phonons, one should form all possible pairing of (phonon) noise lines. ${ }^{23}$ Different contributions can be represented by Feynman graphs such as those shown in Fig. 4. Each solid line represents either the propagator of state 1 [thin (red) line] or that of state 2 [thick (green) line]. These propagators have the form

$$
G_{1,2}\left(t, t^{\prime}\right)=\exp \left( \pm \frac{i}{2} \int_{t^{\prime}}^{t} \Omega(\tau) d \tau\right) .
$$

By introducing these propagators, we absorb the phase factors from the vertex [Eq. (18)]. The dashed (blue) lines represent the correlation functions of the transverse noise $D\left(t, t^{\prime}\right)=\left\langle T\left(u_{\perp}(t) u_{\perp}\left(t^{\prime}\right)\right)\right\rangle$. Each vertex carries the factor $i$ at the upper line and $-i$ at the lower line. Integration over all time arguments must be performed in chronological order at the upper line and in antichronological order at the lower line. In the case of fast not strong noise, the main contribution to the occupation numbers comes from the graphs without crossing or overlapping of the phonon lines. An example of a graph without overlapping is shown in Fig. 3. In comparison with elementary graphs without phonon line overlap-

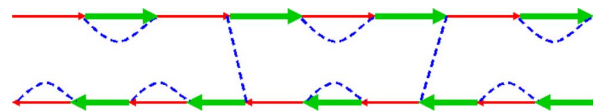

FIG. 3. (Color online) A typical graph without phonon line crossings dominantly contributing to the survival and transition probabilities.
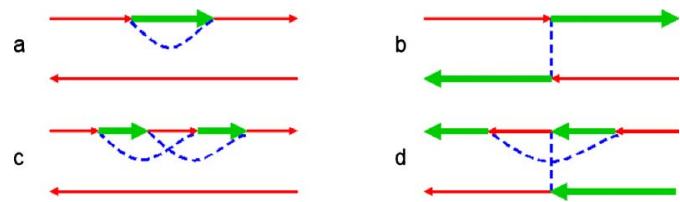

FIG. 4. (Color online) Elementary graphs. [(a) and (b)] without phonon line crossing; [(c) and (d)] with phonon line crossing.

ping or crossing [Figs. 4(a) and 4(b)], the contributions of the graphs containing overlapping or crossing [Figs. 4(c) and 4(d)] have additional small factors of the order of $\left\langle u_{\perp}^{2}\right\rangle \tau_{n}^{2}$ and can be neglected if the transverse noise is moderately strong. Indeed, the time interval between the ends of each phonon lines is about $\tau_{n}$.

To derive the master equation, we consider a set of graphs differing in the occupation numbers $N_{\alpha}(t+\Delta t)$ from those for $N_{\alpha}(t)$, with $\Delta t$ satisfying the following strong inequality: $\tau_{n}$ $\ll \Delta t \ll\left(\left\langle u_{\perp}^{2}\right\rangle \tau_{n}\right)^{-1}$. First, there are graphs with phonon lines connecting the interval $(t, t+\Delta t)$ with the interval $\left(t_{0}, t\right)$. Their contribution can be neglected since it is relatively proportional to a small ratio $\tau_{n} / \Delta t$. The contribution of $k$ nonoverlapping or intersecting noise lines inside the interval $(t, t+\Delta t)$ is proportional to $\left(\left\langle u_{\perp}^{2}\right\rangle \tau_{n} \Delta t\right)^{k} \ll 1$. Therefore, the dominant contribution to the set comes from graphs containing exactly zero or one line inside the interval $(t, t+\Delta t)$. Note that it is a coarse-grain description: the master equation is invalid at time scale $\tau_{n}$ and shorter. The graphical equation connecting $N_{\alpha}(t+\Delta t)$ with $N_{\alpha}(t)$ is shown in Fig. 5.

To find their analytical expression, we calculate the contribution of the three-elementary subgraphs for $\alpha=1$ shown in Fig. 6.

They read

$$
\begin{aligned}
& \Gamma_{1}=\int_{t}^{t+\Delta t} d t_{1} \int_{t}^{t+\Delta t} d t_{2}\left\langle u_{\perp}\left(t_{1}\right) u_{\perp}\left(t_{2}\right)\right\rangle \exp \left(i \int_{t_{2}}^{t_{1}} \Omega(\tau) d \tau\right) \\
& \approx 2 \pi\left\langle u_{\perp} u_{\perp}\right\rangle_{\Omega(t)} \Delta t, \\
& \Pi_{1}=-\int_{t}^{t+\Delta t} d t_{1} \int_{t_{1}}^{t+\Delta t} d t_{2}\left\langle u_{\perp}\left(t_{1}\right) u_{\perp}\left(t_{2}\right)\right\rangle \exp \left(-i \int_{t_{2}}^{t_{1}} \Omega(\tau) d \tau\right) \\
& \approx-\Delta t \int_{-\infty}^{0}\left\langle u_{\perp}(\tau) u_{\perp}(0)\right\rangle e^{-i \Omega(t) \tau} d \tau, \\
& \Pi_{2}=-\int_{t}^{t+\Delta t} d t_{1} \int_{t}^{t_{1}} d t_{2}\left\langle u_{\perp}\left(t_{1}\right) u_{\perp}\left(t_{2}\right)\right\rangle \exp \left(-i \int_{t_{2}}^{t_{1}} \Omega(\tau) d \tau\right) \\
& \approx-\Delta t \int_{0}^{\infty}\left\langle u_{\perp}(\tau) u_{\perp}(0)\right\rangle e^{-i \Omega(t) \tau} d \tau .
\end{aligned}
$$
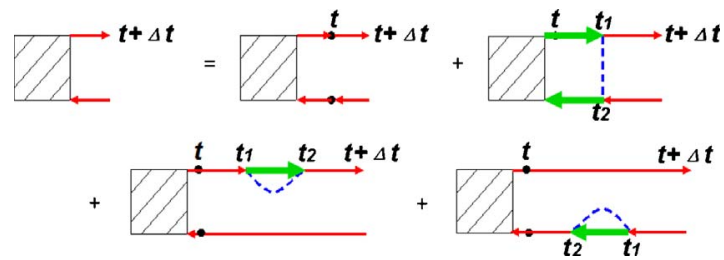

FIG. 5. (Color online) Graphic equation connecting $N_{\alpha}(t+\Delta t)$ and $N_{\alpha}(t)$. 


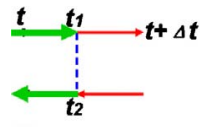

$\Gamma_{1}$

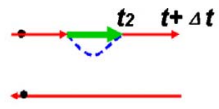

$\Pi_{1}$

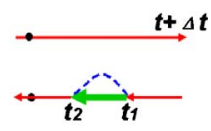

$\Pi$
FIG. 6. (Color online) Three elementary graphs with one phonon line in the interval $t, t+\Delta t$.

In this calculation, we used the fastness of the noise $\left(\tau_{n}\right.$ $\left.\ll \Delta t, \tau_{n} \ll \sqrt{\dot{\Omega}}\right)$ to substitute the integral in the exponent by $\Omega(t)\left(t_{1}-t_{2}\right)$ and to extend the integration over the difference $t_{1}-t_{2}$ to infinite limits. The first graph in Fig. 6 connects $N_{1}(t+\Delta t)$ to $N_{2}(t)$, and two other graphs connect $N_{1}(t+\Delta t)$ to $N_{1}(t)$. Different signs in contributions (22)-(24) are associated with the fact that the vertex at the upper line contains a factor of $-i$, whereas it acquires the factor of $+i$ at the lower line.

Collecting all contributions together, we arrive at the master equations (15) and (16). We rewrite them in a unified form:

$$
\begin{aligned}
\frac{d N_{1}}{d t}= & 2 \pi\left\{N_{2}\left[\theta(\Omega)\left\langle\eta \eta^{\dagger}\right\rangle_{\Omega}+\theta(-\Omega)\left\langle\eta^{\dagger} \eta\right\rangle_{\Omega}\right]\right. \\
& \left.-N_{1}\left[\theta(-\Omega)\left\langle\eta \eta^{\dagger}\right\rangle_{-\Omega}+\theta(\Omega)\left\langle\eta^{\dagger} \eta\right\rangle_{-\Omega}\right]\right\}_{\Omega=\Omega(t)},
\end{aligned}
$$

where $\theta(x)$ is the step function equal to 1 at positive and 0 at negative arguments. The master equation looks simpler when the variable $s_{z}=\frac{N_{1}-N_{2}}{2}=N_{1}-\frac{1}{2}$ is used:

$$
\begin{aligned}
\frac{d s_{z}}{d t}= & 2 \pi\left\{-s_{z}\left[\left\langle\eta \eta^{\dagger}\right\rangle_{|\Omega|}+\left\langle\eta^{\dagger} \eta\right\rangle_{-|\Omega|}\right]\right. \\
& \left.-\frac{1}{2}(\operatorname{sgn} \Omega)\left[\left\langle\eta \eta^{\dagger}\right\rangle_{|\Omega|}-\left\langle\eta^{\dagger} \eta\right\rangle_{-|\Omega|}\right]\right\}_{\Omega=\Omega(t)} .
\end{aligned}
$$

Master equation (25) can be treated as application of the Fermi golden rule to the transition between the two levels separated by an instantaneous frequency $\Omega(t)$. The Fermi golden rule or the first Born approximation at a fixed moment of time can be applied, since the frequency variation during the noise correlation is small and the perturbation caused by the noise in the corresponding stationary problem is weak. The perturbation theory is valid if $\sqrt{\left\langle u_{\perp}^{2}\right\rangle} \ll|\Omega(t)|$. Within the accumulation time interval, the instantaneous frequency is of the same order of magnitude as the spectral width of the noise: $|\Omega(t)| \sim \tau_{n}^{-1}$. The same inequality ensures that the frequency exceeds the width of the levels and the change of frequency due to the interaction with the noise.

Thus, we have proved by microscopic calculation that the quantum coherence is negligible for the time evolution of the occupation numbers. Now, the question about the influence of the longitudinal noise on the master equation is in order. First, we demonstrate that correlations of the type $\left\langle u_{\|}(t) u_{\|}\left(t^{\prime}\right)\right\rangle$ do not change the master equation. Indeed, let us consider the influence of the longitudinal noise on the difference between $N_{\alpha}(t+\Delta t)$ and $N_{\alpha}(t)$. In analogy with the case of the transverse noise, the contribution of one longitudinal

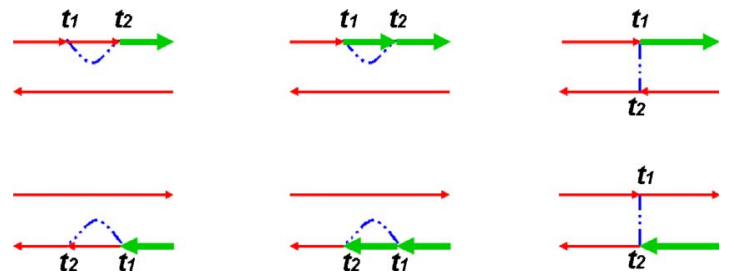

FIG. 7. (Color online) Graphs containing mixed noise correlator (dash-dotted line) and responsible for the LZ gap renormalization.

phonon line inside the interval $(t, t+\Delta t)$ must be taken into account. This contribution does not depend on preceding evolution of the system just because it contains only propagators whose time arguments are confined by the interval $(t, t+\Delta t)$. Therefore, it is the same as it would be in the absence of the transverse noise. Since the longitudinal noise does not produce transitions in the absence of the transverse noise, the total contribution of three graphs of Fig. 6 for longitudinal noise is zero. Direct calculation confirms this statement.

\section{RENORMALIZATION OF THE LANDAU-ZENER GAP}

The problem of mixed correlations between transverse and longitudinal noise is more subtle. The line of mixed correlation starts at one state, to say 1, and ends at another one (2) as shown in Fig. 7. The self-energy part associated with the graphs of the Fig. 7 reads

$$
\begin{aligned}
\Pi_{m i x}= & |1\rangle\langle 2| \exp \left(-i \int_{t_{0}}^{t} \Omega(\tau) d \tau\right)\left[\int_{-\infty}^{0}\left\langle u_{\|}\left(t^{\prime}\right) u_{\perp}(0)\right\rangle d t^{\prime}\right. \\
& \left.-\int_{0}^{\infty}\left\langle u_{\perp}\left(t^{\prime}\right) u_{\|}(0)\right\rangle d t^{\prime}\right]+ \text { H.c. }
\end{aligned}
$$

This operator has the same form as the operator $\Delta \sigma_{x}$ in the interaction representation. Thus, at a time scale much longer than $\tau_{n}$, the mixed correlation renormalizes the LZ gap to a value

$$
\widetilde{\Delta}=\Delta+i \int_{0}^{\infty}\left\langle\left[u_{\perp}\left(t^{\prime}\right), u_{\|}(0)\right]\right\rangle d t^{\prime} .
$$

For the transformation of the integrals in Eq. (27) into the integral in Eq. (28), we employed the time-translation invariance: $\left\langle u_{\|}(-t) u_{\perp}(0)\right\rangle=\left\langle u_{\|}(0) u_{\perp}(t)\right\rangle$. Thus, the statement that one can neglect the action of the transverse noise within the LZ time interval is not completely correct: it is legitimate to neglect transverse-transverse correlations, but the mixed correlations can significantly change the LZ gap up to turning it into zero and changing its sign.

The commutator entering Eq. (28) does not depend on the phonon occupation numbers, i.e., on temperature. It is instructive to express the renormalization of the LZ gap in terms of the phonon model [Sec. II, Eqs. (5), (7), and (8)]:

$$
\widetilde{\Delta}-\Delta=-\frac{1}{V} \sum_{\mathbf{q}} \frac{g_{\|}(\mathbf{q}) g_{\perp}(\mathbf{q})}{\omega_{\mathbf{q}}} .
$$




\section{LONGITUDINAL NOISE}

Next, we consider the action of the purely longitudinal noise within the LZ time interval. For this problem, the transverse noise will be ignored. We start with the classical longitudinal noise to have an idea when it produces substantial changes. The noise is classical if the phonon occupation numbers are large (high temperature), but the noise still may be weak or moderate if the coupling functions $\lambda_{\alpha}(\mathbf{q})$ are small enough. If the noise is classical, the proper diagonal Hamiltonian is $H_{0}=\left(-\frac{\Omega(t)}{2}+u_{\|}\right) \sigma_{z}+H_{b}$, whereas the nondiagonal part is $V=\Delta \sigma_{x}$. In the interaction representation, the nondiagonal part acquires the following form:

$$
V_{I}(t)=\Delta\left(|1\rangle\langle 2| \exp \left(i \int_{t_{0}}^{t}\left(\Omega-2 u_{\|}\right) d \tau\right)+\text { H.c. }\right) \text {. }
$$

Calculation of the transition probability is very similar to that considered above [see Eq. (20) and Fig. 2], but the vertices correspond to $V_{I}(t)$ given by Eq. (30) (we will call them $\Delta$ vertices), and instead of connecting pairs of noise amplitudes, it is necessary to calculate average of a product $\Pi_{j} \exp \left[ \pm 2 i \int_{t_{0}}^{t_{j}} u_{\|}\left(\tau_{j}\right) d \tau_{j}\right]$. Number of the signs - in the exponent is equal to the number of signs + . Therefore, the dependence on the initial moment of time $t_{0}$ vanishes. The Gaussian statistics allows to calculate the average of the product:

$$
\begin{aligned}
& \left\langle\prod_{j} \exp \left[ \pm 2 i \int_{t_{0}}^{t_{j}} u_{\|}\left(\tau_{j}\right) d \tau_{j}\right]\right\rangle \\
& =\exp \left\{-2^{2 k-1} \int_{t_{0}}^{t_{1}} d \tau_{1} \cdots \int_{t_{0}}^{t_{2 k}} d \tau_{2 k}\right. \\
& \left.\quad \times \sum_{C}\left[ \pm\left\langle u_{\|}\left(j_{1}\right) u_{\|}\left(j_{2}\right)\right\rangle\left\langle u_{\|}\left(j_{3}\right) u_{\|}\left(j_{4}\right)\right\rangle+\cdots\right]\right\},
\end{aligned}
$$

where summation is performed over all possible divisions of arguments $\tau_{j}$ into pairs. Each correlator vanishes if the modulus of corresponding time difference $\left|\tau-\tau^{\prime}\right|$ exceeds $\tau_{n}$. The substantial range of integration over the remaining variable $\left(\tau+\tau^{\prime}\right) / 2$ is about $\tau_{L Z}=\Delta / \dot{\Omega}$. Therefore, the order of magnitude of the number obtained in the exponent [Eq. (31)] after integration is $\sim\left(\left\langle u_{\|}^{2}\right\rangle \tau_{n} \tau_{L Z}\right)^{k}$. This value must be of the order or larger than 1 to ensure a significant change of the transition probability by the longitudinal noise. This requirement is equivalent to the inequality $\left\langle u_{\|}^{2}\right\rangle \gtrsim \dot{\Omega} /\left(\Delta \tau_{n}\right) \gg \dot{\Omega}$. For the transverse noise, the analog criterion is much softer: ${ }^{11}$ $\left\langle u_{\perp}^{2}\right\rangle \gtrsim \dot{\Omega}$.

The quantum longitudinal noise does not commute with itself at different moments of time. Therefore, it must be included into the nondiagonal Hamiltonian. Then, besides $\Delta$ vertices, one should consider the noise vertices. Thus, the graphs contain triangular vertices symbolizing a transition between states 1 and 2 and each containing factor $\Delta$, the two-state propagators $G\left(t, t^{\prime}\right)$ defined by Eq. (21), and dotted lines symbolizing the correlators of the longitudinal noise $D_{\|}\left(t, t^{\prime}\right)=\left\langle T u_{\|}(t) u_{\|}\left(t^{\prime}\right)\right\rangle$, which are connected to the two-state propagators by the vertices carrying factors $\pm i$. We obtain the same estimate considering the contribution of an elementary graph with one noise, line. However, for the fast longitudinal noise, it is possible to find not only an estimate but also a system of integral equations for the transition amplitudes. These equations are linear, but they are numerous and their kernel contains a complex combination of parabolic cylinder functions. Their analysis is now in progress. Therefore, their derivation is transferred to the Appendix.

\section{SOLUTION OF THE MASTER EQUATION AND NOISE DIAGNOSTIC}

The master equation (26) allows an explicit solution:

$$
\begin{aligned}
s_{z}(t)= & s_{z}\left(t_{0}\right) \exp \left(-\int_{t_{0}}^{t} f(\tau) d \tau\right) \\
& +\int_{t_{0}}^{t} g\left(t^{\prime}\right) \exp \left(-\int_{t^{\prime}}^{t} f(\tau) d \tau\right) d t^{\prime},
\end{aligned}
$$

where $\quad f(t)=2 \pi\left(\left\langle\eta \eta^{\dagger}\right\rangle_{|\Omega(t)|}+\left\langle\eta^{\dagger} \eta\right\rangle_{-|\Omega(t)|}\right) \quad$ and $\quad g(t)=-\pi$ $\times[\operatorname{sgn} \Omega(t)]\left(\left\langle\eta \eta^{\dagger}\right\rangle_{|\Omega(t)|}-\left\langle\eta^{\dagger} \eta\right\rangle_{-\mid \Omega(t)}\right)$. For classical noise, $g(t)=0$, and Eq. (32) reproduces the result obtained in Ref. 11. At zero temperature, $\left\langle\eta^{\dagger} \eta\right\rangle_{-|\Omega(t)|}=0$ and $g(t)=-\frac{1}{2}$ $\times[\operatorname{sgn} \Omega(t)] f(t)$. In these two cases, the measurement of occupation numbers or $s_{z}(t)$ gives direct information on spectral power of noise. In the classical case, we find $4 \pi\left\langle\eta \eta^{\dagger}\right\rangle_{|\Omega(t)|}=-\frac{d}{d t} \ln \left|s_{z}\right|$; in the purely quantum case $(T=0)$, the relationship is slightly more complicated: $2 \pi\left\langle\eta \eta^{\dagger}\right\rangle_{\mid \Omega(t)} \mid$ $=-\frac{d}{d t} \ln \left|s_{z}+\frac{\operatorname{sgn} \Omega(t)}{2}\right|$. In the general case, it is possible to find both spectral functions $f(t)$ and $g(t)$ by performing two series of measurements with different initial states. Thus, the twostate system is an ideal noise analyzer.

Next, we consider regimes of very fast and very slow (adiabatic) frequency sweepings. In the regime of fast sweeping $\left\langle\eta \eta^{\dagger}\right\rangle \ll \dot{\Omega}$, the perturbation theory for Eq. (32) is valid. Indeed, the integral $\int_{t_{0}}^{t} f(\tau) d \tau$ can be rewritten in terms of spectral power as follows:

$$
\int_{t_{0}}^{t} f(\tau) d \tau=2 \pi \dot{\Omega}^{-1} \int_{\Omega\left(t_{0}\right)}^{\Omega(t)}\left(\left\langle\eta \eta^{\dagger}\right\rangle_{|\omega|}+\left\langle\eta^{\dagger} \eta\right\rangle_{-|\omega|}\right) d \omega .
$$

The integral in the right-hand side of Eq. (33) reaches its maximum value, equal to the average square fluctuation $\left\langle\eta \eta^{\dagger}+\eta^{\dagger} \eta\right\rangle$ at $\Omega\left(t_{0}\right)=-\infty$ and $\Omega(t)=+\infty$. If the condition of fast sweeping is satisfied, the exponent in Eq. (32) can be expanded into a series over small noise parameter $\gamma_{n}=\left\langle\eta \eta^{\dagger}\right.$ $\left.+\eta^{\dagger} \eta\right\rangle / \dot{\Omega}$. The variation $\Delta s_{z}(t)=s_{z}(t)-s_{z}\left(t_{0}\right)$ is small at any time. In the leading approximation, it reads

$$
\begin{aligned}
\Delta s_{z}(t)= & -2 \pi \dot{\Omega}^{-1} \int_{\Omega\left(t_{0}\right)}^{\Omega(t)}\left(\left\langle\eta \eta^{\dagger}\right\rangle_{|\omega|}\left(s_{z}\left(t_{0}\right)+\frac{\operatorname{sgn} \omega}{2}\right)\right. \\
& \left.+\left\langle\eta^{\dagger} \eta\right\rangle_{-|\omega|}\left(s_{z}\left(t_{0}\right)-\frac{\operatorname{sgn} \omega}{2}\right)\right] d \omega .
\end{aligned}
$$

In the opposite regime of slow (adiabatic) sweeping, the noise parameter $\gamma_{n}$ is large. In this case, the exponents in Eq. (32) vary very rapidly allowing asymptotic calculation of $s_{z}$. 
However, in the adiabatic regime, it is simpler to start directly with the master equation (26). Neglecting the time derivative in it, we find the adiabatic solution

$$
s_{z}(t)=\frac{g(t)}{f(t)}=-\frac{\operatorname{sgn} \Omega(t)}{2} \frac{\left\langle\eta \eta^{\dagger}\right\rangle_{|\Omega(t)|}-\left\langle\eta^{\dagger} \eta\right\rangle_{-|\Omega(t)|}}{\left\langle\eta \eta^{\dagger}\right\rangle_{|\Omega(t)|}+\left\langle\eta^{\dagger} \eta\right\rangle_{-}|\Omega(t)|} .
$$

If the photon bath is in equilibrium with temperature $T$, Eq. (35) implies $s_{z}(t)=-\tanh \frac{\Omega(t)}{2 T}$. As it could be expected, at slow sweeping, the two-state system adiabatically accepts the equilibrium population with the temperature of the bath. This conclusion shows that in the case of the quantum noise, one must be more careful with the asymptotic behavior of the time-dependent frequency than in the genuine LZ problem or even in the analog problem with the classical noise. In the latter problems, the linear approximation for $\Omega(t)=\dot{\Omega} t$ was satisfactory. However, this approximation may be invalid for the quantum noise if the sweeping stops before the frequency $\Omega(t)$ reaches the spectral width of the noise. In the opposite case, the value $s_{z}(t)$ saturates after $t=\tau_{a c c}$. In the classical adiabatic case, $s_{z}(t)$ becomes zero after a short time $\tau_{t r}=\left(\left\langle\left|\eta_{\perp}\right|^{2}\right\rangle \tau_{n}\right)^{-1}$. A similar time scale for the longitudinal noise was introduced by Kayanuma and Nakayama. ${ }^{15}$

At the edge of the adiabatic regime, $\gamma_{n} \sim 1$, the fast noise is still moderately strong, i.e., $\sqrt{\left\langle\eta \eta^{\dagger}\right\rangle} \ll \tau_{n}^{-1}$. It means that, when the noise becomes strong, $\sqrt{\left\langle\eta \eta^{\dagger}\right\rangle} \gtrsim \tau_{n}^{-1}$, the system is already in deeply adiabatic regime. If the phonon bath is in equilibrium, the two-state system is also in equilibrium with the noise. This equilibrium state is established in a timeindependent, but strongly nonlinear, system. The interaction of the two-level system characterized by the timeindependent frequency $\Omega$ with the strong noise renormalizes the frequency and creates a finite width for each level. The situation becomes simpler in the limit of very strong noise, $\sqrt{\left\langle\eta \eta^{\dagger}\right\rangle} \gg \tau_{n}^{-1} \gtrsim \Omega$. In this case, the initial energy difference $\Omega$ between levels can be neglected. The two states become equivalent and their occupation numbers are equal (1/2), i.e., $s_{z}=0$. The same result can be obtained from the fact that, as we already argued, the very strong noise must be classical. Equation (35) can be considered as an interpolation between weak and very strong noise. Therefore, it gives a reasonable description of intermediate regime.

\section{TRANSITIONS IN THE PRESENCE OF THE LANDAU-ZENER GAP AND NOISE}

As we demonstrated earlier, for the fast moderately strong noise, the effective time of the LZ transition due to the regular LZ gap $\tau_{L Z}=\Delta / \dot{\Omega}$ is much less than the accumulation time $\tau_{a c c}=\left(\dot{\Omega} \tau_{n}\right)^{-1}$. Therefore, it is possible to ignore the transverse noise within the LZ time interval $|t| \lesssim \tau_{L Z}$ and to ignore the LZ gap $\Delta$ beyond this interval. In this section, we match the LZ solution modified by longitudinal noise inside the LZ interval with the solution of the problem with the transverse noise and $\Delta=0$ (see Sec. VII) beyond this interval. For this purpose, we choose a time scale $t_{1}$ such that $\tau_{L Z}$ $\ll t_{1} \ll \tau_{a c c}$ and first consider the solution [Eq. (32)] of the master equation in the interval $\left(-\infty,-t_{1}\right)$, then use the LZ solution with longitudinal noise ignoring transverse noise in the interval $\left(-t_{1}, t_{1}\right)$, and again use the solution of the master equation in the interval $\left(t_{1},+\infty\right)$. The next step is to put $t_{1}$ $=0$ for the solution of the master equation and to put $t_{1}=\infty$ for the solution of the LZ equations with longitudinal noise. Limiting values of the solutions at intervals left from $\pm t_{1}$ serve as initial values for solutions at intervals right from them. First, we consider the interval $\left(-\infty,-t_{1}\right)$. For simplification, we accept in the solution [Eq. (32)] $t_{0}=-\infty$. Since $t_{1}$ $\ll \tau_{a c c}$, it can be replaced by 0 in the solution [Eq. (32)] with high precision $\sim t_{1} / \tau_{a c c}$. Thus, at the left edge of the interval $\left(-t_{1}, t_{1}\right)$, we find

$$
\begin{aligned}
s_{z}\left(-t_{1}\right) \simeq & s_{z}^{(-)}=\exp \left(-\int_{-\infty}^{0} f(\tau) d \tau\right) s_{z}(-\infty) \\
& +\int_{-\infty}^{0} g\left(t^{\prime}\right) \exp \left(-\int_{t^{\prime}}^{0} f(\tau) d \tau\right) d t^{\prime} .
\end{aligned}
$$

This value can be treated as an initial condition $s_{z}^{(-)}$at $t=-\infty$ for the LZ problem with the longitudinal noise. If the solution of this problem is known, the value $s_{z}^{(+)}$at $t=+\infty$ can be calculated. The information necessary to make this calculation effective is the knowledge of two numbers if there is no coherence in the initial system. The density matrix $\rho^{(+)}$at $t=+\infty$ is obviously a linear function of the initial density matrix $\rho^{(-)}$. There exists a linear $4 \times 4$ matrix $\Lambda$ performing this transformation:

$$
\rho_{\alpha \beta}^{(+)}=\Lambda_{\alpha \beta, \mu \nu} \rho_{\mu \nu}^{(-)}
$$

The requirement that $\operatorname{Tr} \rho^{(+)}=1$ if $\operatorname{Tr} \rho^{(-)}=1$ implies the following equation: $\Lambda_{\alpha \alpha, \mu \nu}=\delta_{\mu \nu}$. If $\rho_{12}^{(-)}=\rho_{21}^{(-)}=0$, Eq. (37) results in following relationship between $s_{z}^{(+)}$and $s_{z}^{(-)}$:

$$
s_{z}^{(+)}=\left(\Lambda_{1}+\Lambda_{2}\right) s_{z}^{(-)}+\left(\Lambda_{1}-\Lambda_{2}\right),
$$

where we introduced abbreviations $\Lambda_{1}$ and $\Lambda_{2}$ for $\Lambda_{11,11}$ and $\Lambda_{22,22}$, respectively. If the longitudinal noise is absent or sufficiently weak, the LZ values for $\Lambda_{\alpha}$ are

$$
\Lambda_{1}=\Lambda_{2}=\exp \left(-2 \pi \gamma_{L Z}\right)-\frac{1}{2}
$$

If $\left\langle u_{\|}^{2}\right\rangle \ll \dot{\Omega} /\left(\Delta \tau_{n}\right)$, the longitudinal noise is weak enough to neglect the longitudinal-longitudinal correlations. Still the correlation of the longitudinal and transverse noise can significantly change the effective LZ gap [see Eq. (28)]. The values $\Lambda_{1,2}$ for some specific situations in which the longitudinal noise is substantial can be extracted from the cited works. ${ }^{14,15}$

The value $s_{z}^{(+)}$from Eq. (39) serves, in turn, as initial condition at $t=+0$ for the master equation. Its solution [Eq. (32)] at $t=+\infty$ leads to the following final result: 


$$
\begin{aligned}
s_{z}(+\infty)= & \left(\Lambda_{1}+\Lambda_{2}\right) e^{-2 \pi \gamma_{\perp}} s_{z}(-\infty) \\
& +\left(\Lambda_{1}-\Lambda_{2}\right) e^{-\pi \gamma_{\perp}} \frac{\pi}{\dot{\Omega}} \int_{0}^{\infty}\left(\left\langle\eta \eta^{\dagger}\right\rangle_{\Omega}-\left\langle\eta^{\dagger} \eta\right\rangle_{-\Omega}\right) \\
& \times \exp \left[-\frac{2 \pi}{\dot{\Omega}} \int_{\Omega}^{\infty}\left(\left\langle\eta \eta^{\dagger}\right\rangle_{\omega}+\left\langle\eta^{\dagger} \eta\right\rangle_{-\omega}\right) d \omega\right] \\
& \times\left[( \Lambda _ { 1 } + \Lambda _ { 2 } ) \operatorname { e x p } \left(-\frac{4 \pi}{\dot{\Omega}} \int_{0}^{\Omega}\left(\left\langle\eta \eta^{\dagger}\right\rangle_{\omega}\right.\right.\right. \\
& \left.\left.\left.+\left\langle\eta^{\dagger} \eta\right\rangle_{-\omega}\right) d \omega\right)-1\right] d \Omega .
\end{aligned}
$$

Here, $\gamma_{\perp}=\left\langle u_{\perp}^{2}\right\rangle / \dot{\Omega}$. We remind that the occupation numbers are related to $s_{z}$ as $N_{1,2}=1 / 2 \pm s_{z}$. Below, we write the survival probability for the case when the longitudinallongitudinal correlations can be neglected, $\left\langle u_{\|}^{2}\right\rangle / \Omega \ll\left(\Delta \tau_{n}\right)^{-1}$. In this case, $s_{z}(-\infty)=1 / 2$, the values $\Lambda_{1,2}$ are determined by Eq. (39), and from Eq. (40), we find

$$
\begin{aligned}
& P_{1 \rightarrow 1}=\frac{1}{2}\left[1+e^{-2 \pi \gamma_{\perp}}\left(2 e^{-2 \pi \gamma_{L Z}}-1\right)\right]+\frac{\pi}{\dot{\Omega}} \int_{0}^{\infty}\left(\left\langle\eta \eta^{\dagger}\right\rangle_{\Omega}\right. \\
& \left.-\left\langle\eta^{\dagger} \eta\right\rangle_{-\Omega}\right) \exp \left[-\frac{2 \pi}{\dot{\Omega}} \int_{\Omega}^{\infty}\left(\left\langle\eta \eta^{\dagger}\right\rangle_{\omega}+\left\langle\eta^{\dagger} \eta\right\rangle_{-\omega}\right) d \omega\right] \\
& \times\left[( 2 e ^ { - 2 \gamma _ { L Z } } - 1 ) \operatorname { e x p } \left(-\frac{4 \pi}{\dot{\Omega}} \int_{0}^{\Omega}\left(\left\langle\eta \eta^{\dagger}\right\rangle_{\omega}\right.\right.\right. \\
& \left.\left.\left.+\left\langle\eta^{\dagger} \eta\right\rangle_{-\omega}\right) d \omega\right)-1\right] d \Omega \text {. }
\end{aligned}
$$

To take into account the correlation between the longitudinal and transverse noise, one should replace the LZ gap $\Delta$ in the expression for $\gamma_{L Z}=\Delta^{2} / \dot{\Omega}$ by the renormalized value from Eqs. (28) and (29).

\section{DISCUSSION AND CONCLUSIONS}

In the case of weak transverse noise or very fast sweeping, $\gamma_{\perp} \ll 1$, Eqs. (40) and (41) turn into Eq. (38) and the LZ survival probability, respectively. In the opposite case of the strong transverse noise or slow sweeping, $\gamma_{\perp} \gg 1$, the occupation numbers accept their stationary values at fixed instantaneous frequency independent of the value of LZ parameter $\gamma_{L Z}$. The classical noise corresponds to large phonon occupation numbers $n_{\mathbf{q}}$. In this case, the operators $\eta$ and $\eta^{\dagger}$ commute; all terms containing commutators $\left\langle\eta \eta^{\dagger}\right\rangle_{\Omega}-\left\langle\eta^{\dagger} \eta\right\rangle_{-\Omega}$ can be neglected. Then, theory reproduces the result for classical fast noise. ${ }^{11}$ It is instructive to compare Eq. (41) with the exact survival probability for $T=0$ obtained in the recent article by Wubs et al. ${ }^{16}$ At zero temperature, the average value $\left\langle\eta^{\dagger}(t) \eta\left(t^{\prime}\right)\right\rangle$ as well as its Fourier transform turns into zero. This fact allows us to calculate the integrals in Eq. (41). A more physically visible way of obtaining the same result is to keep in mind that there is no live phonon at $T=0$ and only spontaneous emission of phonons is possible. Therefore, if initially only the lower state was populated, the phonon cannot be emitted before the level crossing. This consideration immediately gives $s_{z}^{(-)}=s_{z}(-\infty)=\frac{1}{2}$ and $s_{z}^{(+)}=\frac{1}{2}\left(2 e^{-2 \gamma_{L Z}}-1\right)$. Employing general equation (32), we find the value $s_{z}(+\infty)$ $=\frac{1}{2}\left[2 \exp \left(-2 \pi\left(\gamma_{L Z}+\gamma_{\perp}\right)\right)-1\right]$ and the survival probability

$$
\begin{aligned}
P_{1 \rightarrow 1}= & \exp \left(-2 \pi\left(\gamma_{L Z}+\gamma_{\perp}\right)\right) \\
= & \exp \left\{-\frac{2 \pi}{\dot{\Omega}}\left[\left(\Delta-\frac{1}{V} \sum_{\mathbf{q}} \frac{g_{\|}(\mathbf{q}) g_{\perp}(\mathbf{q})}{\omega_{\mathbf{q}}}\right)^{2}\right.\right. \\
& \left.\left.+\left\langle\eta_{\perp}(0) \eta_{\perp}^{\dagger}(0)\right\rangle\right]\right\} .
\end{aligned}
$$

This result with precision of notations coincides with the exact result by Wubs et al., ${ }^{16}$ Eqs. (6)-(8), obtained without any limitations to the strength of noise and ratios of characteristic time scales. Surprisingly, the multiphonon processes as well as the longitudinal-longitudinal noise correlations do not contribute at all to the survival and transition probabilities even at very high noise intensity. At zero temperature, such a high noise level can be reached only by enhancement of the coupling amplitudes. Though large coupling amplitudes are physically implausible, as a mathematical model, they are absolutely legitimate. The fact that these highintensity processes do not play a role in the transitions supports our speculations that the master equation range of validity may be broader than what follows from our derivation.

Our theory is relevant to molecular magnets, first of all because the condition of the noise fastness is perfectly satisfied in the experiment. Indeed, the highest magnetic field rate used in the experiments with $\mathrm{Fe}_{8}$ and $\mathrm{Mn}_{12}$ was $10^{3} \mathrm{G} / \mathrm{s} .{ }^{25,26}$ This rate corresponds to $\dot{\Omega}=10^{10} \mathrm{~s}^{-2}$. The lowest temperature used in the cited measurements was about $0.05 \mathrm{~K}$. The dimensionless ratio of the value $\sqrt{\dot{\Omega}}$ to the smaller of the noise spectral widths is $\hbar \sqrt{\dot{\Omega}} / T \sim 10^{-5}$.

The renormalization of the gap due to the correlation between longitudinal and transverse noise (see Sec. V) allows resolving at least qualitatively two puzzles which appeared in the experimental studies of nanomagnets. The first of them relates to the selection rules at transitions. A simple but rather general model accepted in theory of nanomagnets ${ }^{28}$ describes them by a model Hamiltonian $H=-a S_{z}^{2}+b S_{x}^{2}$, where $a$ and $b$ are positive anisotropy constants $(a>b)$. Such a Hamiltonian has nondegenerate spectrum, but at large $S$, the lowest energy levels characterized with a dominant projection of spin $\pm M$ are only slightly split. Being placed into the time-dependent magnetic field, these states change their energy and cross each other. The LZ transitions at such crossings are due to the term $b S_{x}^{2}$ in the Hamiltonian. Therefore, only transitions between levels with the same parity of $M$ are allowed by theory in contradiction with the experiment. The renormalization of the gap lifts this contradiction because the spin-phonon interaction introduces terms such as $u_{x z} S_{x} S_{z}$. They result in nonzero renormalized gap between the states with different parities of $M$ and zero initial gap.

The second puzzle is the isotopic variation of the LZ gap $\Delta .{ }^{27}$ Wernsdorfer et al. ${ }^{29}$ have found a significant effect at isotopic substitution of $\mathrm{Fe}_{58}$ by $\mathrm{Fe}_{57}$ and $\mathrm{H}$ by $\mathrm{D}$ in the mol- 
ecule $\mathrm{Fe}_{8}$. The isotopic dependence looks puzzling if the origin of the gap is electronic spin-orbit interaction, but it is quite natural in the gap renormalization due to phonons.

In principle, our theory can explain the temperature dependence of the hysteresis curve in molecular magnets. The molecular hysteresis curve displays clearly pronounced steps at definite, temperature independent values of the magnetic field. ${ }^{25,26,28}$ They are identified with the LZ transitions at crossings of Zeeman levels belonging to different $M$. The hysteresis curve becomes temperature independent at temperature below $0.4 \mathrm{~K}$, but it strongly narrows at comparatively low temperature about $1.5-3 \mathrm{~K}$. The application of our theory to the problem of the hysteresis curve and relaxation requires several additional steps. The first is establishing of the spin-phonon Hamiltonian. Its simplest form is $H_{s-p h}=\Lambda_{\alpha \beta, \gamma \delta} u_{a \beta} S_{\gamma} S_{\delta}$ for interaction with acoustic phonons or $H_{s-p h}=\Lambda_{\alpha, \beta \gamma} u_{\alpha} S_{\beta} S_{\gamma}$ for interaction with optic phonons. The coefficients $\Lambda_{\alpha \beta, \gamma \delta}$ can be obtained from the measurements of magnetization curves under the pressure and shear deformation. To our knowledge, there are no such experimental data so far. We do not know how to extract the opticphonon coupling constants $\Lambda_{\alpha, \beta \gamma}$ from experimental data. Both these sets can be found by numerical calculations for a single molecule. Keeping in mind a large number of atoms in it, it is not a simple computational problem. We plan to derive a kind of effective media approach. However, even if the spin-phonon coupling would be known, it is necessary to translate them into the language of the two-level (spin 1/2) system near each diabatic Zeeman level crossing. This procedure does not require additional knowledge of parameters, but it requires work and time. We will give here a rough estimate of the noise intensity. The transverse noise is very weak for transitions with a large change of the spin projection, for example, from +10 to -10 , since the standard coupling of the deformations to spin can change the spin projection only by $\pm 1, \pm 2$. Therefore, the transverse noise linear in phonon operators appears only in combination with highorder perturbation caused by the biaxial anisotropy $b$. For transitions with the small change of the spin projection, the transverse noise is of the same order of magnitude as the longitudinal one. Assuming that the coefficients $\Lambda_{\alpha \beta, \gamma \delta}$ have the same order of magnitude as $a$ and $b$, intensity can be estimated from the known value of magnetic anisotropy energy $a \simeq 0.06 \mathrm{~K}$ (for $\mathrm{Fe}_{8}$ ) and the statistical weight of phonons with frequency smaller than the anisotropy energy $\sim\left(a / E_{D}\right)^{3}$, where $E_{D}$ is the Debye energy. An additional factor $a / E_{D}$ comes from the square of coupling function $\left|g_{\mathbf{q}}\right|^{2}$, which is proportional to $q$ at small wave vectors. Thus, $\left\langle u_{\|}^{2}\right\rangle \sim a^{2}\left(a / E_{D}\right)^{4} \sqrt{\frac{m}{M}} \ll a^{2}=\left(\hbar \omega_{g}\right)^{2}$. This inequality shows that the condition of moderately strong noise is well satisfied for the acoustic noise. Numerical estimate of the value $\left\langle u_{\|}^{2}\right\rangle / \hbar^{2} \dot{\Omega}$ shows that it is small for the magnetic sweeping rate from 1 to $1000 \mathrm{G} / \mathrm{s}$ used in the experiment. Thus, the acoustic noise scarcely can explain the strong temperature dependence of the hysteresis curve. More plausibly, low-frequency intramolecular oscillations are responsible for this phenomenon. However, there is no doubt that the thermal noise becomes important at a temperature of a few Kelvins and that the noise is fast and quantum. Though the longitudinal noise does not produce transitions between diabatic levels, it is effective for transitions between adiabatic levels. ${ }^{14}$

In conclusion, we derived the master equations for moderately strong fast quantum transverse noise. We obtained its solution at any moment of time. We demonstrated that the action of the regular LZ transition matrix element and the longitudinal-longitudinal noise correlation is limited by the LZ time scale, whereas the action of the transverse noise is accumulated during much longer accumulation time. We showed that the mixed longitudinal-transverse noise correlation leads to the renormalization of the LZ gap $\Delta$. In the limiting case of adiabatic transverse noise, the two-state system adiabatically follows its stationary state at an instantaneous value of frequency independent of the value of LZ parameter. The separation of time scales allows us to derive exact transition probability with the LZ gap, with longitudinal and transverse noise taken into account simultaneously. The transition probabilities depend explicitly on the noise commutator reflecting the quantum nature of the noise. It plays an important role especially in the adiabatic regime. In the extreme quantum regime at zero temperature, our result coincides with the exact result by Wubs et al. ${ }^{16}$ We argued that the strong-noise effects such as multiphonon processes and change of frequency appear only in the adiabatic regime for the fast noise and do not substantially change the transition probability.

\section{ACKNOWLEDGMENTS}

Our thanks are due to N. Sinitsyn, Y. Gefen, and A. Abanov for useful discussions and B. Dobrescu for participation in the initial stage of this work and a valuable remark. We also thank M. Wubs for useful correspondence. This work was supported by the DOE under the Grant No. DEFG02-06ER46278 and partly by the NSF under the Grant No. DMR-0321572. V.L.P. acknowledges the hospitality and support of the Max Planck Institut für Komplexen Sytemen, Dresden, during the Workshop on Mesoscopics, AugustSeptember 2006.

\section{APPENDIX: EQUATIONS FOR FAST LONGITUDINAL NOISE}

In this appendix, we derive a system of differential equations for the longitudinal noise acting together. The characteristic interval in which both these factors are effective is the LZ time $\tau_{L Z}$. The action of transverse noise during this time can be neglected. First, we prove that the contribution of the irreducible graphs, ${ }^{24}$ which do not connect different branches of the Keldysh contour, is zero. Indeed the remote ends of such graphs are separated by the time interval of the order of $\tau_{n} \ll \tau_{L Z}$. Therefore, it is possible to integrate over the time difference at a fixed "center of time" or "slow time" as we did in the case of the transverse noise. In contrast to the latter case, for longitudinal noise, the integral does not depend on slow time, since the longitudinal noise vertex in the interaction representation does not contain timedependent phase factor (it connects identical states of the two-state system). By the same reason, it is the same for 

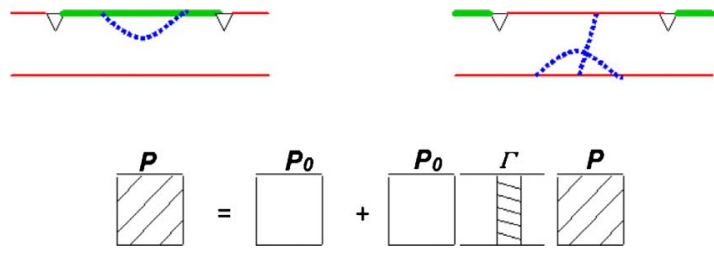

FIG. 8. (Color online) Graphs containing the longitudinal noise only. Triangles correspond to the LZ gap $\Delta$. (a) Graphs with one noise loop. In the slow time scale, they are equivalent to addition of a constant energy. (b) Graphs with a line connecting Keldysh branches. (c) General graphic equation for $P$. See explanation in the text.

states 1 and 2. Therefore, in a slow time scale much longer than $\tau_{n}$ (but much less than $\tau_{L Z}$ ), the contribution of such graphs is proportional to unit operator for the two-state system and can be completely ignored. The irreducible noise graphs connecting different branches of Keldysh contour form a four-pole vertex (see Fig. 8), which, by the same reason, does not depend on slow time and connects identical states at each branch of the Keldysh contour. We denote this vertex by $\Gamma$.

Since the number of $\Delta$ vertices between vertices $\Gamma$ is arbitrary, we need to extend the number of amplitudes in consideration. Namely, we define transition amplitudes $P_{\alpha \beta, \alpha_{0} \beta_{0}}$ $\left(t, t_{0}\right)$ as the average value of the operator $|\alpha\rangle\langle\beta|$ at the moment $t$ if the density matrix for two-state system at the moment $t_{0}$ was $\left|\alpha_{0}\right\rangle\left\langle\beta_{0}\right|$. In the accepted approximation, these amplitudes obey a system of linear integral equations:

$$
\begin{aligned}
P_{\alpha \beta, \alpha_{0} \beta_{0}}\left(t, t_{0}\right)= & P_{\alpha \beta, \alpha_{0} \beta_{0}}^{(0)}\left(t, t_{0}\right)-\Gamma \int_{t_{0}}^{t} P_{\alpha \beta, \alpha^{\prime} \beta^{\prime}}\left(t, t^{\prime}\right) P_{\alpha^{\prime} \beta^{\prime}, \alpha_{0} \beta_{0}}^{(0)} \\
& \times\left(t^{\prime}, t_{0}\right) d t^{\prime} .
\end{aligned}
$$

Here, $P_{\alpha \beta, \alpha_{0} \beta_{0}}^{(0)}\left(t, t_{0}\right)$ denotes the transition amplitude in the absence of noise. The validity of Eq. (A1) is limited by moderately strong noise. Otherwise, the amplitudes $P_{\alpha \beta, \alpha_{0} \beta_{0}}$ $\left(t, t_{0}\right)$ vary significantly at a time scale $\tau_{n}$. In this case, more complicated equations with a nonlocal in time kernel $\Gamma$ and amplitudes depending on four time arguments must be used.
${ }^{1}$ L. D. Landau, Phys. Z. Sowjetunion 2, 46 (1932).

${ }^{2}$ C. Zener, Proc. R. Soc. London, Ser. A 137, 696 (1932).

${ }^{3}$ E. C. G. Stückelberg, Helv. Phys. Acta 5, 369 (1932).

${ }^{4}$ A. J. Legget, S. Chakravarty, A. T. Dorsey, M. P. A. Fisher, A. Garg, and W. Zwerger, Rev. Mod. Phys. 59, 1 (1987).

${ }^{5}$ U. Weiss, Quantum Dissipative Systems (World Scientific, Singapore, 1998).

${ }^{6}$ A. Izmalkov, M. Grajcar, E. Il'ichev, N. Oukhanski, Th. Wagner, H.-G. Meyer, W. Krech, M. H. S. Amin, Alec Brink van den Maassen, and A. M. Zagoskin, Europhys. Lett. 65, 844 (2004); I. Chiorescu, P. Bertet, K. Samba, Y. Nakayama, C. J. P. M. Harmans, and J. E. Mooij, Nature (London) 431, 159 (2004).

${ }^{7}$ M. Kusunoki, Phys. Rev. B 20, 2512 (1979).

${ }^{8}$ Y. Kayanuma, J. Phys. Soc. Jpn. 54, 2037 (1985).

${ }^{9}$ The noise is called transverse if it produces transitions between the two states in the absence of regular transition matrix element.

${ }^{10}$ Y. Kayanuma, J. Phys. Soc. Jpn. 53, 108 (1984).

${ }^{11}$ V. L. Pokrovsky and N. A. Sinitsyn, Phys. Rev. B 67, 144303 (2003).

${ }^{12}$ V. L. Pokrovsky and S. Scheidl, Phys. Rev. B 70, 014416 (2004).

${ }^{13}$ Y. Gefen, E. Ben-Jacob, and A. O. Caldeira, Phys. Rev. B 36, 2770 (1987).

${ }^{14}$ P. Ao and J. Rammer, Phys. Rev. B 43, 5397 (1991).

${ }^{15}$ Y. Kayanuma and H. Nakayama, Phys. Rev. B 57, 13099 (1998).

${ }^{16}$ M. Wubs, K. Saito, S. Kohler, P. Hänggi, and Y. Kayanuma, Phys. Rev. Lett. 97, 200404 (2006).

${ }^{17}$ The result for survival probability obtained (Ref. 16) is a consequence of the theorem proposed by Brandobler and Elser (Ref.
18) as a hypothesis and proved rigorously by Dobrescu and Sinitsyn (Ref. 19). Wubs et al., have found the same method independently, but a little later. Important steps toward the proof of the Brandobler-Elser hypothesis were made by A. V. Shytov, Phys. Rev. B 71, 085301 (2005); N. A. Sinitsyn, J. Phys. A 37, 10691 (2004); M. V. Volkov and V. N. Ostrovsky, J. Phys. B, 37 4069 (2004); 38, 907 (2005).

${ }^{18}$ S. Brandobler and V. Elser, J. Phys. A 26, 1211 (1993).

${ }^{19}$ B. Dobrescu and N. A. Sinitsyn, J. Phys. B 39, 1253 (2006).

${ }^{20}$ A. B. Migdal, Sov. Phys. JETP 7, 996 (1958).

${ }^{21}$ L. V. Keldysh, Zh. Eksp. Teor. Fiz. 47, 1515 (1964).

${ }^{22}$ J. Schwinger, J. Math. Phys. 2, 407 (1961).

${ }^{23}$ The Wick pairing ensures that the numbers of the state alternations on the upper and the lower lines are either both even or both odd. Therefore, the occupation numbers depend only on diagonal matrix elements of the initial density matrix.

${ }^{24}$ Following the quantum field tradition, we call a graph irreducible if it cannot be separated into two disconnected parts (which are not connected by a noise line).

${ }^{25}$ W. Wernsdorfer and R. Sessoli, Science 284, 133 (1999).

${ }^{26}$ J. R. Friedman, M. P. Sarachik, J. Tejada, and R. Ziolo, Phys. Rev. Lett. 76, 3830 (1996).

${ }^{27}$ We are thankful to N. Sinitsyn who attracted our attention to this opportunity.

${ }^{28}$ D. Gatteschi, R. Sessoli, and J. Villain, Molecular Nanomagnets (Oxford University Press, New York, 2006).

${ }^{29}$ W. Wernsdorfer, R. Sessoli, A. Ganeschi, D. Gatteschi, and A. Cornia, Europhys. Lett. 50, 552 (2000). 